\title{
DIGITALCOMMONS
}

5-1-2014

\section{Change Point Estimation for Pareto Type-II Model}

Gyan Prakash

S. N. Medical College, Agra, U. P., India, ggyanji@yahoo.com

Follow this and additional works at: http://digitalcommons.wayne.edu/jmasm

Part of the Applied Statistics Commons, Social and Behavioral Sciences Commons, and the Statistical Theory Commons

\section{Recommended Citation}

Prakash, Gyan (2014) "Change Point Estimation for Pareto Type-II Model," Journal of Modern Applied Statistical Methods: Vol. 13 : Iss. 1 , Article 22.

DOI: $10.22237 /$ jmasm/1398918060

Available at: http://digitalcommons.wayne.edu/jmasm/vol13/iss1/22

This Regular Article is brought to you for free and open access by the Open Access Journals at DigitalCommons@WayneState. It has been accepted for inclusion in Journal of Modern Applied Statistical Methods by an authorized editor of DigitalCommons@WayneState. 


\section{Change Point Estimation for Pareto Type-II Model}

Gyan Prakash

S. N. Medical College

Agra, U.P., India

Some Bayes estimators of the change point for the Pareto Type-II model under right item failure-censoring scheme are proposed. The Bayes estimators are obtained here in two cases, the first is when one parameter is known and second when both parameters are considered as the random variable. The performances of the procedures are illustrated by simulation technique.

Keywords: $\quad$ Change point, Pareto Type-II model, Bayes estimation

\section{Introduction}

The Pareto distribution and its close relatives provide a flexible family of fattailed distributions, which may be used as a model for income distribution of higher income group and in socio-economic studies. This distribution has played important role in variety of other problems such as size of cities and firms, business mortality, service time in queuing system. It is often used as a model for analyzing areas including city population distribution, stock price fluctuation, oil field locations and military areas.

It has been found to be suitable for approximating the right tails of distribution with positive skewness. Pareto distribution has a decreasing failure rate, so it has often been used for model survival after some medical procedures (the ability to survive for a longer time appears to increase, the longer one survives after certain medical procedures).

Harries (1968) used this distribution in determining times of maintenance service while Dyer (1981) found that two-parameter Pareto distribution transformation is equivalent to the two-parameter exponential distribution. Madi $\&$ Raqab (2004) discussed about the forecasting of the temperatures records by

Gyan Prakash is an Assistant Professor in the Department of Community Medicine.

Email himat: ggyanji@yahoo.com. 


\section{CHANGE POINT ESTIMATION FOR PARETO TYPE-II MODEL}

Pareto distribution. Singh et al (2007) discussed about different types of testestimation for the Pareto Model. The length of Bayes prediction limits have been obtained recently by Prakash \& Singh (2013) for the Pareto model. Panahi \& Asadi (2011) presented stress-Strength model for a Lomax distribution. Some inferences regarding the Lomax distribution under the generalized order statistics has discussed by Moghadam et al. (2012). Nasiri \& Hosseini (2012) presented Bayesian and classical statistical inferences for Lomax model based on record values. Recently, Al-Zahrani \& Al-Sobhi (2013) presents some parameter estimation for Lomax distribution under general progressive censoring criterion.

The probability density function of the considered Pareto Type-II model is given as

$$
f(x ; \sigma, \theta)=\theta \sigma^{\theta}(x+\sigma)^{-(\theta+1)} ; x \geq 0, \theta>0, \sigma>0
$$

Here, $\theta$ is the shape parameter and $\sigma$ is the scale parameter. The proposed Pareto Type-II model is the result of mixture of the Exponential distribution with the parameter $\alpha$, and the exponential scale parameter $\alpha$ is distributed as a Gamma with parameters $\theta$ and $\sigma$.

This article discusses the Bayes estimation of change point for Pareto TypeII model. The Bayes estimator has been obtained under the right item failure censoring criteria in two cases: the first is when the scale parameter is known and second when both parameters are considered as the random variable. A numerical study was carried out for illustration of the procedures in next section by MCMC technique.

\section{The Change Point}

In order to obtain information on their endurance, manufactured items such as mechanical or electronic components are often put to life tests and life times are observed periodically. Physical systems manufacturing the items are often subject to random fluctuations. It may happen that at some point of time, there is a change in the parameter. The objective of study is to find out when and where this change has started occurring, which is called the change point inference problem.

Bayesian model may play an important role in the study of such change point estimation problem and have been studied by Broemeling \& Tsurumi (1987), Jani \& Pandya (1999), Ebrahimi \& Ghose (2001), Goldenshluger, et al. (2006). Pandya \& Jadav (2010) presents Bayesian estimation of change point in mixture of left truncated exponential and degenerate distribution. Some Bayes estimation 


\section{GYAN PRAKASH}

of shift point in Poisson model was presented by Srivastava (2012). Recently, Pandya (2013) presented Bayes estimation of auto regressive model with change point.

Consider a sequence of independent random sample of size $n(\geq 3)$ such as $x_{1}, x_{2}, \ldots, x_{m-1}, x_{m}, x_{m+1}, \ldots, x_{n}$ from the considered model with survival function $\psi_{1}(t)$ at time $t(>0)$ but later it is found that there is a change in the system at some point of time $m$ and it is reflected in the sequence after the observation $x_{m}$ by the change in the survival function. The probability density function and survival function of the first $m$ observations $x_{1}, x_{2}, \ldots, x_{m}$ are given from model (1) as:

$$
f\left(x_{i} ; \sigma, \theta_{1}\right)=\theta_{1} \sigma^{\theta_{1}}\left(x_{i}+\sigma\right)^{-\left(\theta_{1}+1\right)} ; x_{i} \geq 0, \theta_{1}>0, \sigma>0, i=1,2, \ldots, m
$$

and

$$
\psi_{1}(t)=\sigma^{\theta_{1}}(t+\sigma)^{-\theta_{1}} ; \mathrm{t}>0, \theta_{1}>0, \sigma>0
$$

Similarly, the probability density function and survival function of remaining $(n-m)$ components $x_{m+1}, x_{m+2}, \ldots, x_{n}$ are

$$
\begin{aligned}
& f\left(x_{i} ; \sigma, \theta_{2}\right)=\theta_{2} \sigma^{\theta_{2}}\left(x_{i}+\sigma\right)^{-\left(\theta_{2}+1\right)} \\
& x_{i} \geq 0, \theta_{2}>0, \sigma>0, i=m+1, \mathrm{~m}+2, \ldots, n
\end{aligned}
$$

and

$$
\psi_{2}(t)=\sigma^{\theta_{2}}(t+\sigma)^{-\theta_{2}} ; \mathrm{t}>0, \theta_{2}>0, \sigma>0
$$

In life testing, the observations usually occur in ordered manner such that the weakest items fail first and then second one and so on. Suppose that $n$ items are put to test under the considered model without replacement and only $k(\leq n)$ items are fully measured, while the remaining $(n-k)$ items are censored. These $(n-k)$ censored items will be ordered separately. This censoring scheme is known as the right item failure-censoring criteria.

The change point criteria was introduced inside the right item-censoring scheme; assume a sequence of ordered independent random sample of size $n$ such as $x_{(1)}, x_{(2)}, \ldots, x_{(k-1)}, x_{(k)}, x_{(k+1)}, \ldots, x_{(n)}$ from the model (1), with the 


\section{CHANGE POINT ESTIMATION FOR PARETO TYPE-II MODEL}

parameters $\theta_{1}$ and $\sigma$. All $n$ items are tested without replacement and first $k$ ordered items are fully measured while remaining $(n-k)$ items are censored. From the first fully measured $k\left(=x_{(1)}, x_{(2)}, \ldots, x_{(k-1)}, x_{(k)}\right)$ items, it is found that there is a change in the system at some point of time $m$ and it is reflected in the sequence after $x_{(m)}(m \leq k)$ by the change in the survival function.

The probability density function of first $m(m \leq, k \leq n)$ random samples $x_{(1)}, x_{(2)}, \ldots, x_{(m)}$ with parameters $\theta_{1}$ and $\sigma$, are

$$
\begin{array}{r}
f\left(x_{(i)} ; \sigma, \theta_{1}\right)=\theta_{1} \sigma^{\theta_{1}}\left(x_{(i)}+\sigma\right)^{-\left(\theta_{1}+1\right)} ; \\
x_{(i)}, \theta_{1}, \sigma>0, i=1,2, \ldots, m(m \leq k, k \leq n) .
\end{array}
$$

The first remaining group of random samples $x_{(m+1)}, x_{(m+2)}, \ldots, x_{(k)}$ with size $(k-m)$ using a considered Pareto model has a probability density function with parameters $\theta_{2}$ and $\sigma$

$$
\begin{aligned}
f\left(x_{(i)} ; \sigma, \theta_{2}\right)=\theta_{2} \sigma^{\theta_{2}}\left(x_{(i)}+\sigma\right)^{-\left(\theta_{2}+1\right)} ; \\
x_{(i)}, \theta_{2}, \sigma>0, i=m+1, \mathrm{~m}+2, \ldots, k(k \geq m, k \leq n) .
\end{aligned}
$$

The last remaining group of random samples $x_{(k+1)}, x_{(k+2)}, \ldots, x_{(n)}$ of size $(n-k)$ distributed again a Pareto model with parameters $\theta_{1}$ and $\sigma$-has the probability density function

$$
\begin{aligned}
& f\left(x_{(i)} ; \sigma, \theta_{1}\right)=\theta_{1} \sigma^{\theta_{1}}\left(x_{(i)}+\sigma\right)^{-\left(\theta_{1}+1\right)} \\
& x_{(i)}, \theta_{1}, \sigma>0, i=k+1, \mathrm{k}+2, \ldots, n(\geq k) .
\end{aligned}
$$

Under the above scenario the likelihood function for the random sample $\underline{x}\left(=x_{(1)}, x_{(2)}, \ldots, x_{(n)}\right)$ is defined as

$$
\begin{aligned}
L\left(\underline{x} \mid \theta_{1}, \theta_{2}, \sigma, m\right) & =\left(\prod_{i=1}^{m} f\left(x_{(i)} ; \sigma, \theta_{1}\right)\right) \cdot\left(\prod_{i=m+1}^{k} f\left(x_{(i)} ; \sigma, \theta_{2}\right)\right) \\
& \cdot \prod_{i=k+1}^{n}\left(1-\int_{0}^{x_{(i)}} f\left(x_{(i)} ; \sigma, \theta_{1}\right) d x_{(i)}\right)
\end{aligned}
$$




\section{GYAN PRAKASH}

$$
\Rightarrow L\left(\underline{x} \mid \theta_{1}, \theta_{2}, \sigma, m\right)=T_{0} \theta_{1}^{m} \theta_{2}^{k-m} \sigma^{\theta_{1}(n-k+m)+\theta_{2}(k-m)} e^{-\theta_{1} T_{1}} e^{-\theta_{2} T_{2}}
$$

where $T_{0}=\prod_{i=1}^{k}\left(x_{(i)}+\sigma\right)^{-1}, \quad T_{1}=\sum_{i=1}^{m} \log \left(x_{(i)}+\sigma\right)+\sum_{i=k+1}^{n} \log \left(x_{(i)}+\sigma\right) \quad$ and $T_{2}=\sum_{i=\mathrm{m}+1}^{k} \log \left(x_{(i)}+\sigma\right)$.

\section{Remark}

1. Substitute $\theta_{1}=\theta=\theta_{2}$ in (7)

$$
L(\underline{x} \mid \theta, \sigma)=T_{0} \quad \theta^{k} \quad \sigma^{n \theta} e^{-\theta T_{3}} ; T_{3}=\sum_{i=1}^{n} \log \left(x_{(i)}+\sigma\right) .
$$

Here, $L(\underline{x} \mid \theta, \sigma)$ shows the likelihood function under the right itemfailure censoring criterion without consideration of change point.

2. Substitute $\theta_{1}=\theta=\theta_{2}$ and $k=n$ in (7) to obtain the likelihood function for complete sample case without consideration of change point.

$$
L(\underline{x} \mid \theta, \sigma)=T_{0}^{*} \theta^{n} \sigma^{n \theta} e^{-\theta T_{3}} ; T_{0}^{*}=\prod_{i=1}^{n}\left(x_{(i)}+\sigma\right)^{-1} .
$$

\section{Change Point Estimation (Scale Parameter Is Known)}

From a Bayesian viewpoint; there is clearly no way in which one can say that one prior is better than other. It is more frequently the case that, that a prior is selected to restrict attention to a given natural family of priors, and one is chosen from that family, which seems to match best with one's personal beliefs. A natural family of conjugate prior for shape parameter $\theta$ is considered here as a Gamma distribution (when scale parameter is known) with probability density function

$$
g(\theta \mid \sigma) \propto \theta^{a-1} e^{-\beta \theta} ; \theta>0, a>0, \beta>0 .
$$

Based on change point criterion the prior density (8) is re-parameterized as 


\section{CHANGE POINT ESTIMATION FOR PARETO TYPE-II MODEL}

$$
g_{j}\left(\theta_{j} \mid \sigma\right) \propto \theta_{j}^{a_{j}-1} e^{-\beta_{j} \theta_{j}} ; \theta_{j}>0, a_{j}>0, \beta_{j}>0, j=1,2 .
$$

A discrete uniform over the set $(1,2, \ldots, k-1)$, is considered as the prior distribution of change point $m$ and defined as

$$
g_{3}(m)=\frac{1}{k-1} .
$$

The joint prior distribution when scale parameter is considered to be known, is defined as

$$
h_{1}\left(\theta_{1}, \theta_{2}, m\right)=g_{1}\left(\theta_{1} \mid \sigma\right) \cdot g_{2}\left(\theta_{2} \mid \sigma\right) \cdot g_{3}(m) \text {. }
$$

The joint posterior density function is now obtained as

$$
\begin{gathered}
\pi_{1}\left(\theta_{1}, \theta_{2}, m \mid \underline{x}\right)=\frac{L\left(\underline{x} \mid \theta_{1}, \theta_{2}, \sigma, \mathrm{m}\right) \cdot h_{1}\left(\theta_{1}, \theta_{2}, m\right)}{\sum_{m} \int_{\theta_{1}} \int_{\theta_{2}} L\left(\underline{x} \mid \theta_{1}, \theta_{2}, \sigma, \mathrm{m}\right) \cdot h_{1}\left(\theta_{1}, \theta_{2}, m\right) d \theta_{2} d \theta_{1}} \\
\Rightarrow \pi_{1}\left(\theta_{1}, \theta_{2}, m \mid \underline{x}\right)=\bar{\sigma} \theta_{1}^{m+\theta_{1}-1} \theta_{2}^{k-m+\theta_{2}-1} e^{-T_{1}^{*} \theta_{1}} e^{-T_{2}^{*} \theta_{2}} ; \\
\text { where } \bar{\sigma}=\left(\sum_{m=1}^{k-1} \Delta\right)^{-1}, \Delta=\left(\frac{\Gamma\left(m+a_{1}\right) \Gamma\left(k-m+a_{2}\right)}{\left(T_{1}^{*}\right)^{m+a_{1}}\left(T_{2}^{*}\right)^{k-m+a_{2}}}\right), T_{1}^{*}=T_{1}+\beta_{1}-(n-k+m)
\end{gathered}
$$

$\log \sigma$ and $T_{2}^{*}=T_{2}+\beta_{2}-(k-m) \log \sigma$.

Hence, the marginal posterior density for change point $m$ is

$$
\pi^{*}(m \mid \underline{x})=\int_{\theta_{1}} \int_{\theta_{2}} \pi_{1}\left(\theta_{1}, \theta_{1}, m \mid \underline{x}\right) d \theta_{2} d \theta_{1}=\bar{\sigma} \Delta
$$

The choice of the loss function may be crucial in Bayesian analysis. It has always been recognized that the most commonly used loss function, squared error loss function (SELF), is inappropriate in many situations. The Bayes estimator of a parameter under SELF is the posterior mean. If SELF is taken as a measure of inaccuracy then the resulting risk is often too sensitive to the assumptions about the behavior of the tail of the probability distribution. To overcome this difficulty, 


\section{GYAN PRAKASH}

a useful asymmetric loss function based on the squared error loss function (ISELF) is defined for any estimate $\hat{\theta}$ corresponding to the parameter $\theta$ as

$$
L(\hat{\theta}, \theta)=\left(\theta^{-1} \partial\right)^{2} ; \partial=\hat{\theta}-\theta
$$

The Bayes estimator of the change point $m$ under ISELF is obtained as

$$
\begin{aligned}
\hat{m}_{I} & =E_{P}\left(m^{-1}\right) / E_{P}\left(m^{-2}\right) \\
& \Rightarrow \hat{m}_{I}=\sum_{m=1}^{k-1}\left(m^{-1} \Delta\right) / \sum_{m=1}^{k-1}\left(m^{-2} \Delta\right) .
\end{aligned}
$$

Here, the suffix $P$ indicates the expectation taken under the posterior density.

When positive and negative errors have different consequences, the use of squared error loss function (SELF) in Bayesian estimation may not be appropriate. In addition, in some estimation problems overestimation is more serious than the underestimation, or vice-versa. To deal with such cases, a useful and flexible class of asymmetric loss function (LINEX loss function (LLF)) is given as

$$
L(\partial)=e^{a \partial}-a \partial-1
$$

The shape parameter of LLF is denoted by ' $a$ '. Negative (positive) value of shape parameter ' $a$ ', gives more weight to overestimation (underestimation) and its magnitude reflect the degree of asymmetry. It is also observed that, for $a=1$, the function is very asymmetric with overestimation being more costly than underestimation. For small values of $|a|$, the LLF is almost symmetric and is not far from the SELF.

Bayes estimator of $m$ under the LLF is obtained as

$$
\begin{aligned}
\hat{m}_{L} & =-\frac{1}{a} \log E_{P}\left\{e^{-a m}\right\} \\
& =-\frac{1}{a} \log \left\{\bar{\sigma} \sum_{m=1}^{k-1}\left(\Delta e^{-a m}\right)\right\} .
\end{aligned}
$$

A close form of both the estimators does not exist. A numerical method is applied for obtaining the values of their estimates. 


\section{Change Point Estimation (Both Parameter Unknown)}

In the case of when both the parameters $\theta$ and $\sigma$ are unknown for the considered Pareto model, there does not exists any joint conjugate prior. Assume that the prior beliefs about the parameters $\theta$ and $\sigma$ are independent. The natural family of conjugate priors for parameters $\theta$ and non-informative prior for parameter $\sigma$ are considered independently here. The non-informative prior of the parameter $\sigma$ is the limiting form of the appropriate natural conjugate prior. The joint prior distribution when both parameters are unknown is defined as

$$
g(\theta, \sigma)=g(\theta \mid \sigma) \cdot h(\sigma) ; h(\sigma)=\frac{1}{\sigma}, \sigma>0
$$

The prior distribution $g(\theta \mid \sigma)$ is given in equation (8). The likelihood function in present case is redefined as

$$
\begin{aligned}
L\left(\underline{x} \mid \theta_{1}, \theta_{2}, \sigma, m\right) & =\left(\prod_{i=1}^{m} f\left(x_{(i)} ; \sigma, \theta_{1}\right)\right) \\
& \cdot\left(\prod_{i=m+1}^{k} f\left(x_{(i)} ; \sigma, \theta_{2}\right)\right) \cdot \prod_{i=k+1}^{n}\left(1-\int_{0}^{x_{(i)}} f\left(x_{(i)} ; \sigma, \theta_{1}\right) d x_{(i)}\right) \\
\Rightarrow L\left(\underline{x} \mid \theta_{1}, \theta_{2}, \sigma, m\right) & =T_{0} \theta_{1}^{m} \theta_{2}^{k-m} \mathrm{e}^{\theta_{1}(n-k+m) \log \sigma} \mathrm{e}^{\theta_{2}(k-m) \log \sigma} e^{-\theta_{1} T_{1}} e^{-\theta_{2} T_{2}} .
\end{aligned}
$$

The joint prior density for the parameters $\theta_{1}, \theta_{2}, \sigma$ and $m$ is written as

$$
h_{2}\left(\theta_{1}, \theta_{2}, \sigma, m\right)=g_{1}\left(\theta_{1} \mid \sigma\right) \cdot g_{2}\left(\theta_{2} \mid \sigma\right) \cdot h(\sigma) \cdot g_{3}(m) \text {. }
$$

Hence, the joint posterior density is obtained as

$$
\begin{aligned}
& \pi_{2}\left(\theta_{1}, \theta_{2}, \sigma, m \mid \underline{x}\right)=\frac{L\left(\underline{x} \mid \theta_{1}, \theta_{2}, \sigma, m\right) \cdot h_{2}\left(\theta_{1}, \theta_{2}, \sigma, m\right)}{\sum_{m} \iint_{\sigma} \int_{\theta_{1}} L\left(\underline{x} \mid \theta_{1}, \theta_{2}, \sigma, m\right) \cdot h_{2}\left(\theta_{1}, \theta_{2}, \sigma, m\right) d \theta_{2} d \theta_{1} d \sigma} \\
& \Rightarrow \pi_{2}\left(\theta_{1}, \theta_{2}, \sigma, m \mid \underline{x}\right)=\overline{\bar{\sigma}} \frac{T_{0}}{\sigma} \theta_{1}^{m+a_{1}-1} \theta_{2}^{k-m+a_{2}-1} e^{-T_{1}^{*} \theta_{1}} e^{-T_{2}^{*} \theta_{2}} ;
\end{aligned}
$$




\section{GYAN PRAKASH}

where $\overline{\bar{\sigma}}=\left(\sum_{m=1}^{k-1} \bar{\Delta}\right)^{-1}$ and $\bar{\Delta}=\int_{\sigma} \frac{\Delta T_{0}}{\sigma} d \sigma$.

Hence, the marginal posterior density for the change point $m$ is

$$
\pi^{* *}(m \mid \underline{x})=\int_{\sigma} \int_{\theta_{1}} \int_{\theta_{2}} \pi_{2}\left(\theta_{1}, \theta_{1}, \sigma, m \mid \underline{x}\right) d \theta_{2} d \theta_{1} d \sigma=\overline{\bar{\sigma}} \bar{\Delta}
$$

and, the Bayes estimator under ISELF and LLF for the change point $m$ are obtained as

$$
\hat{\hat{m}}_{I}=\sum_{m=1}^{k-1}(\bar{\Delta} / m) / \sum_{m=1}^{k-1}\left(\bar{\Delta} / m^{2}\right)
$$

and

$$
\hat{\hat{m}}_{L}=-\frac{1}{a} \log \left\{\overline{\bar{\sigma}} \sum_{m=1}^{k-1}\left(e^{-a m} \bar{\Delta}\right)\right\} .
$$

\section{Numerical Analysis}

\section{One Parameter Known Case}

To assess and study the properties of the Bayes estimator for the change point $m$, a simulation study was performed. The random samples were generated as:

Generate $\theta_{i} ; i=1,2$; through prior density $g_{i}\left(\theta_{i}\right) ; i=1,2$; for the given values of prior parameters $\alpha_{i}, \beta_{i} ; i=1,2$; as $\left(\alpha_{i}, \beta_{i}\right)=(0.25,0.50),(4,2)$, $(9,3) ; i=1,2$. The selections of prior parametric values meet the criterion that the prior variance should be unity.

Using generated values of $\theta_{i} ; i=1,2$; and $\sigma=0.50,1.00,1.50,3.00$; generate 10,000 random samples of size $n=15$ by using the model (2) and (3).

The values of the Bayes estimate $\hat{m}_{I}$ under the ISELF have been obtained and presented them in the Table 1 , for selected set of censored sample size $k=04,06,08,10$. 
CHANGE POINT ESTIMATION FOR PARETO TYPE-II MODEL

Table 1. Bayes Estimate of $m$ under ISELF (Scale Parameter Known)

\begin{tabular}{cccccccc}
\multicolumn{2}{c}{$n=15$} & \multicolumn{5}{c}{$k \downarrow$} \\
\cline { 1 - 4 }$\sigma$ & $(\alpha, \beta) \downarrow$ & & $\mathbf{4}$ & $\mathbf{6}$ & $\mathbf{8}$ & $\mathbf{1 0}$ & $\mathbf{1 5}$ \\
\hline 0.50 & $0.25,0.50$ & & 3.7066 & 3.7177 & 3.7251 & 3.7326 & 3.7400 \\
0.50 & 04,02 & & 3.6908 & 3.7019 & 3.7092 & 3.7166 & 3.7239 \\
0.50 & 09,03 & & 3.3381 & 3.3481 & 3.3549 & 3.3615 & 3.3681 \\
& & & & & & & \\
1.00 & $0.25,0.50$ & & 3.7336 & 3.7447 & 3.7522 & 3.7597 & 3.7672 \\
1.00 & 04,02 & & 3.7052 & 3.7163 & 3.7236 & 3.7311 & 3.7385 \\
1.00 & 09,03 & & 3.6519 & 3.6627 & 3.6700 & 3.6773 & 3.6846 \\
& & & & & & \\
1.50 & $0.25,0.50$ & & 3.7371 & 3.7483 & 3.7558 & 3.7633 & 3.7707 \\
1.50 & 04,02 & & 3.7177 & 3.7289 & 3.7363 & 3.7436 & 3.7510 \\
1.50 & 09,03 & & 3.6906 & 3.7016 & 3.7091 & 3.7164 & 3.7238 \\
& & & & & & \\
3.00 & $0.25,0.50$ & 3.4849 & 3.4953 & 3.5024 & 3.5094 & 3.5165 \\
3.00 & 04,02 & 3.4701 & 3.4806 & 3.4875 & 3.4944 & 3.5012 \\
3.00 & 09,03 & 3.4088 & 3.4189 & 3.4257 & 3.4325 & 3.4393 \\
\hline
\end{tabular}

Table 1 shows that when censored sample size $k$ increases, the magnitude of the estimate increases, but increment in magnitude is nominal (robust). A similar trend also noted when scale parameter $\sigma$ increases, however for large value of $\sigma(>1.5)$ the magnitude of the estimate decreases. The opposite trend has been seen when set of prior parameter increases.

Using above considered set of parametric values with $a=0.25,0.50,1.00,2.00$; (shape parameter of LLF) the magnitude of the Bayes estimate under LLF have been obtained and present in the Table 2, only for $a=0.25,1.00$. 


\section{GYAN PRAKASH}

Table 2. Bayes Estimate of $m$ under LLF (Scale Parameter Known)

\begin{tabular}{ccccccc}
\multicolumn{2}{c}{$n=15, a=0.25$} & \multicolumn{5}{c}{$k \downarrow$} \\
\hline$\sigma$ & $(\alpha, \beta) \downarrow$ & $\mathbf{4}$ & $\mathbf{6}$ & $\mathbf{8}$ & $\mathbf{1 0}$ & $\mathbf{1 5}$ \\
\hline 0.50 & $0.25,0.50$ & 3.3060 & 3.3159 & 3.3226 & 3.3292 & 3.3358 \\
0.50 & 04,02 & 3.2778 & 3.2877 & 3.2942 & 3.3007 & 3.3073 \\
0.50 & 09,03 & 2.9531 & 2.9620 & 2.9678 & 2.9737 & 2.9797 \\
& & & & & & \\
1.00 & $0.25,0.50$ & 3.2839 & 3.2937 & 3.3002 & 3.3069 & 3.3135 \\
1.00 & 04,02 & 3.2699 & 3.2798 & 3.2863 & 3.2927 & 3.2992 \\
1.00 & 09,03 & 3.2698 & 3.2795 & 3.2860 & 3.2926 & 3.2991 \\
& & & & & & \\
1.50 & $0.25,0.50$ & 3.2331 & 3.2426 & 3.2491 & 3.2556 & 3.2621 \\
1.50 & 04,02 & 3.2192 & 3.2289 & 3.2353 & 3.2417 & 3.2480 \\
1.50 & 09,03 & 3.1623 & 3.1717 & 3.1780 & 3.1842 & 3.1906 \\
& & & & & & \\
3.00 & $0.25,0.50$ & 2.9560 & 2.9648 & 2.9708 & 2.9768 & 2.9827 \\
3.00 & 04,02 & 2.9433 & 2.9522 & 2.9581 & 2.9639 & 2.9699 \\
3.00 & 09,03 & 2.8913 & 2.8999 & 2.9057 & 2.9115 & 2.9173
\end{tabular}

\begin{tabular}{ccccccc}
\multicolumn{2}{c}{$n=15, a=1.00$} & \multicolumn{5}{c}{$k \downarrow$} \\
\hline$\sigma$ & $(\alpha, \beta) \downarrow$ & $\mathbf{4}$ & $\mathbf{6}$ & $\mathbf{8}$ & $\mathbf{1 0}$ & $\mathbf{1 5}$ \\
\hline 0.50 & $0.25,0.50$ & 4.0109 & 4.0228 & 4.0309 & 4.0390 & 4.0470 \\
0.50 & 04,02 & 3.9937 & 4.0058 & 4.0137 & 4.0216 & 4.0295 \\
0.50 & 09,03 & 3.9231 & 3.9348 & 3.9425 & 3.9504 & 3.9583 \\
& & & & & & \\
1.00 & $0.25,0.50$ & 3.7329 & 3.7440 & 3.7515 & 3.7590 & 3.7665 \\
1.00 & 04,02 & 3.7010 & 3.7121 & 3.7194 & 3.7269 & 3.7343 \\
1.00 & 09,03 & 3.3344 & 3.3444 & 3.3510 & 3.3577 & 3.3644 \\
& & & & & & \\
1.50 & $0.25,0.50$ & 3.7030 & 3.7140 & 3.7214 & 3.7289 & 3.7363 \\
1.50 & 04,02 & 3.6871 & 3.6982 & 3.7056 & 3.7129 & 3.7202 \\
1.50 & 09,03 & 3.6869 & 3.6980 & 3.7054 & 3.7127 & 3.7201 \\
& & & & & & \\
3.00 & $0.25,0.50$ & 2.7579 & 2.7661 & 2.7716 & 2.7772 & 2.7828 \\
3.00 & 04,02 & 2.7460 & 2.7544 & 2.7598 & 2.7652 & 2.7707 \\
3.00 & 09,03 & 2.6975 & 2.7055 & 2.7109 & 2.7163 & 2.7217 \\
\hline
\end{tabular}




\section{CHANGE POINT ESTIMATION FOR PARETO TYPE-II MODEL}

Table 2 shows that when shape parameter $\sigma$ increases, the magnitude of the estimator decreases (except for large prior parametric value). An increasing trend in the magnitude of the estimate also is also noted when ' $a$ ' increases but the increment in magnitude is robust. Others properties are similar to ISELF.

\section{When Both Parameters Unknown}

When both parameters are considered as a random variable, a simulation study was carried out to study the properties of Bayes estimators of change point.

Similarly, a 10,000 random sample of size $n=15$ was generated. The Bayes estimate of $m$ under the ISELF and LLF were obtained and are presented in Tables 3-4 respectively for different selected set of values.

Table 3. Bayes Estimate of $m$ under ISELF (Both Parameter Unknown)

\begin{tabular}{ccccccc}
\multicolumn{2}{c}{$n=15$} & \multicolumn{5}{c}{$k \downarrow$} \\
\hline$\sigma$ & $(\alpha, \beta) \downarrow$ & $\mathbf{4}$ & $\mathbf{6}$ & $\mathbf{8}$ & $\mathbf{1 0}$ & $\mathbf{1 5}$ \\
\hline 0.50 & $0.25,0.50$ & 3.3640 & 3.3741 & 3.3808 & 3.3877 & 3.3944 \\
0.50 & 04,02 & 3.3497 & 3.3599 & 3.3664 & 3.3731 & 3.3798 \\
0.50 & 09,03 & 3.2223 & 3.2320 & 3.2384 & 3.2448 & 3.2513 \\
& & & & & & \\
1.00 & $0.25,0.50$ & 3.6074 & 3.6183 & 3.6254 & 3.6327 & 3.6399 \\
1.00 & 04,02 & 3.5766 & 3.5873 & 3.5945 & 3.6017 & 3.6088 \\
1.00 & 09,03 & 3.2905 & 3.3003 & 3.3068 & 3.3134 & 3.3200 \\
& & & & & & \\
1.50 & $0.25,0.50$ & 3.6150 & 3.6258 & 3.6331 & 3.6403 & 3.6477 \\
1.50 & 04,02 & 3.5996 & 3.6105 & 3.6176 & 3.6248 & 3.6319 \\
1.50 & 09,03 & 3.5982 & 3.6090 & 3.6161 & 3.6233 & 3.6304 \\
& & & & & & \\
3.00 & $0.25,0.50$ & 3.6787 & 3.6896 & 3.6971 & 3.7045 & 3.7118 \\
3.00 & 04,02 & 3.6630 & 3.6740 & 3.6813 & 3.6886 & 3.6959 \\
3.00 & 09,03 & 3.5995 & 3.6103 & 3.6174 & 3.6246 & 3.6318 \\
\hline
\end{tabular}




\section{GYAN PRAKASH}

Table 4. Bayes Estimate of $m$ under LLF (Both Parameter Known)

\begin{tabular}{|c|c|c|c|c|c|c|}
\hline \multicolumn{2}{|c|}{$n=15, a=0.25$} & \multicolumn{5}{|c|}{$k \downarrow$} \\
\hline$\sigma$ & $(\alpha, \beta) \downarrow$ & 4 & 6 & 8 & 10 & 15 \\
\hline 0.50 & $0.25,0.50$ & 3.4309 & 3.4411 & 3.4480 & 3.4550 & 3.4617 \\
\hline 0.50 & 04,02 & 3.4162 & 3.4265 & 3.4333 & 3.4401 & 3.4469 \\
\hline 0.50 & 09,03 & 3.3558 & 3.3658 & 3.3725 & 3.3792 & 3.3859 \\
\hline 1.00 & $0.25,0.50$ & 3.2678 & 3.2775 & 3.2841 & 3.2907 & 3.2972 \\
\hline 1.00 & 04,02 & 3.2398 & 3.2496 & 3.2560 & 3.2624 & 3.2689 \\
\hline 1.00 & 09,03 & 3.1907 & 3.2002 & 3.2066 & 3.2130 & 3.2194 \\
\hline 1.50 & $0.25,0.50$ & 3.2046 & 3.2141 & 3.2205 & 3.2269 & 3.2334 \\
\hline 1.50 & 04,02 & 3.1907 & 3.2002 & 3.2066 & 3.2130 & 3.2194 \\
\hline 1.50 & 09,03 & 2.9189 & 2.9277 & 2.9335 & 2.9392 & 2.9452 \\
\hline 3.00 & $0.25,0.50$ & 2.4142 & 2.4214 & 2.4262 & 2.4312 & 2.4361 \\
\hline 3.00 & 04,02 & 2.4039 & 2.4112 & 2.4159 & 2.4207 & 2.4255 \\
\hline 3.00 & 09,03 & 2.3614 & 2.3684 & 2.3732 & 2.3779 & 2.3826 \\
\hline \multicolumn{2}{|c|}{$n=15, a=1.00$} & \multicolumn{5}{|c|}{$k \downarrow$} \\
\hline$\sigma$ & $(\alpha, \beta) \downarrow$ & 4 & 6 & 8 & 10 & 15 \\
\hline 0.50 & $0.25,0.50$ & 3.7918 & 3.8031 & 3.8108 & 3.8183 & 3.8260 \\
\hline 0.50 & 04,02 & 3.7756 & 3.7871 & 3.7946 & 3.8020 & 3.8095 \\
\hline 0.50 & 09,03 & 3.7088 & 3.7199 & 3.7272 & 3.7347 & 3.7421 \\
\hline 1.00 & $0.25,0.50$ & 3.5352 & 3.5458 & 3.5528 & 3.5599 & 3.5671 \\
\hline 1.00 & 04,02 & 3.5201 & 3.5308 & 3.5378 & 3.5448 & 3.5517 \\
\hline 1.00 & 09,03 & 3.5199 & 3.5305 & 3.5375 & 3.5446 & 3.5516 \\
\hline 1.50 & $0.25,0.50$ & 3.2398 & 3.2496 & 3.2560 & 3.2624 & 3.2689 \\
\hline 1.50 & 04,02 & 3.1908 & 3.2005 & 3.2068 & 3.2131 & 3.2195 \\
\hline 1.50 & 09,03 & 3.0107 & 3.0194 & 3.0207 & 3.0328 & 3.0475 \\
\hline 3.00 & $0.25,0.50$ & 2.6585 & 2.6665 & 2.6717 & 2.6772 & 2.6826 \\
\hline 3.00 & 04,02 & 2.6472 & 2.6552 & 2.6603 & 2.6657 & 2.6710 \\
\hline 3.00 & 09,03 & 2.6003 & 2.6080 & 2.6133 & 2.6185 & 2.6237 \\
\hline
\end{tabular}




\section{CHANGE POINT ESTIMATION FOR PARETO TYPE-II MODEL}

The behavior of $\hat{\hat{m}}_{I}$ was shown to be similar as compare to $\hat{m}_{I}$ under ISELF. It is also noted that the magnitude of the estimator $\hat{\hat{m}}_{l}$ increases as $\sigma$ increases for all selected parametric set of values. Further, the magnitude of the estimate of $\hat{\hat{m}}_{I}$ is closer than the estimate of $\hat{m}_{l}$ except for large value of $\sigma$.

All properties of estimator $\hat{\hat{m}}_{L}$ were similar as compared to $\hat{m}_{L}$ under LLF. For small values of ' $a$ ', the magnitude of estimate of $\hat{m}_{L}$ is wider than $\hat{\hat{m}}_{L}$ for all considered values of $\sigma$ (except for $\sigma=0.50$ ). For large values of ' $a$ ', the magnitude of estimate of $\hat{\hat{m}}_{L}$ becomes narrower than $\hat{m}_{L}$ for all considered values of $\sigma$ (except for $\sigma=1.00$ ). Other properties are the same, as in the case of a known shape parameter.

\section{Remark}

In the case when the censored sample size $r=15$, the censoring criterion reduces to the complete sample size criterion and, hence, all results are valid for the complete sample case.

\section{References}

Al-Zahrani, B. \& Al-Sobhi, M. (2013). On parameters estimation of Lomax distribution under general progressive censoring. Journal of Quality and Reliability Engineering, 2013: 1-7.

Broemeling, L. D. \& Tsurumi, H. (1987). Econometrics and structural change. New York: Marcel Dekker.

Dyer, D. (1981). Structural probability bounds for the strong Pareto law. Canadian Journal Statistics, 9: 71-77.

Ebrahimi, N. \& Ghosh, S. K. (2001). Bayesian and frequentist methods in change-point problems. In N. Balkrishna \& C. R. Rao (Eds). Handbook of statistics, Vol. 20: Advances in Reliability, (Elsevier), 777-787.

Goldenshluger, A., Tsybakov, A. \& Zeevi, A. (2006). Optimal change point estimation from indirect observations. The Annals of Statistics, 34(1): 350-372.

Harris, C. M. (1968). The Pareto distribution as a queue discipline. Operations Research, 16(2): 307-313. 


\section{GYAN PRAKASH}

Jani, P. N. \& Pandya, M. (1999). Bayes estimation of shift point in left truncated exponential sequence. Communications in Statistics-Theory and Methods, 28(11): 2623-2639.

Madi, M. T. \& Raqab, M. Z. (2004). Bayesian prediction of temperature records using the Pareto model. Environmetrics, 15(7): 701-710.

Moghadam, M. S., Farhad, Y. \& Manoochehr, B. (2012). Inference for Lomax distribution under generalized order statistics. Applied Mathematical Sciences, 6(105): 5241-5251

Nasiri, P. \& Hosseini, S. (2012). Statistical inferences for Lomax distribution based on record values (Bayesian and Classical). Journal of Modern Applied Statistical Methods, 11(1): 179-189.

Panahi, H. \& Asadi, S. (2011). Inference of stress-strength model for a Lomax distribution. World Academy of Science, Engineering and Technology, 55: 275-278.

Pandya, M. (2013). Bayesian estimation of AR (1) with change point under asymmetric loss functions. Statistical Research Letters, 2(2): 53-62.

Pandya, M. \& Jadav, P. (2010). Bayesian estimation of change point in mixture of left truncated exponential and degenerate distribution. Communication in statistics-Theory and Methods, 39(15): 2725-2742.

Prakash, G. \& Singh, D. C. (2013). Bayes prediction intervals for the Pareto model. Journal of Probability and Statistical Science, 11(1): 109-122.

Singh, D. C., Prakash, G. \& Singh, P. (2007). Shrinkage testimators for the shape parameter of Pareto distribution using LINEX loss function. Communications in Statistics-Theory and Methods, 36(4): 741-753.

Srivastava, U. (2012). Bayesian estimation of shift point in Poisson model under asymmetric loss functions. Pakistan Journal of Statistics and Operation Research, 8(1): 31-42. 